\title{
Efficacy and acceptability of outpatient, single-visit, medical abortion up to 59 days of gestation
}

This article was published in the following Dove Press journal:

Open Access Journal of Contraception

23 November 2010

Number of times this article has been viewed

\section{Manisha Agarwal \\ Department of Obstetrics and Gynecology, Mahatma Gandhi Medical College and Research Institute, Pillaiyarkuppam, Pondicherry, India}

Correspondence: Manisha Agarwal B6/2 I 2, Shreenath ji Vihar, Sitapur Road, Nirala Nagar Extension, Lucknow 226020, India

$\mathrm{Tel}+94 \mid 5769829$

Email manishaagarwal60@gmail.com

\begin{abstract}
This study evaluated the efficacy and acceptability of medical abortion by a modified, single-visit treatment regimen for termination of early (up to 59 days) pregnancy. The study population included 160 women. They were vaginally administered misoprostol $600 \mathrm{mg}$ six hours after oral administration of mifepristone $200 \mu \mathrm{g}$. Patients' symptoms were evaluated by telephone, and they were counseled accordingly. Follow-up sonography was performed two weeks later. The reasons patients chose this method were to avoid hospitalization, preference for a natural and noninvasive method, and confidentiality (55\%). Side effects were mainly mild abdominal cramps (45\%) and prolonged bleeding (2.4\%). The success rate of complete abortion was $98.12 \%$, with $1.25 \%$ of these patients needing a second vaginal dose to complete the treatment. The single-visit medical abortion method is acceptable and efficacious for women in developing countries.
\end{abstract}

Keywords: medical abortion, mifepristone, misoprostol, administration, intravaginal

\section{Introduction}

In India, 3.9-6.0 million abortions are conducted annually. However, this number is probably a gross underestimate, given that most abortions are either not reported or are performed illegally. ${ }^{1}$ Termination of pregnancy has been legalized in India under the Medical Termination of Pregnancy (MTP) Act, 1971. Two methods, ie, surgical abortion and medical abortion, are generally preferred for early termination of pregnancy.

Surgical evacuation is the traditional method. However, this approach has disadvantages, including need for admission to hospital, administration of anesthesia, instrumentation, immediate procedural morbidity, absence from work and loss of manpower, and long-term consequences affecting future pregnancy. These disadvantages can be overcome by employing medical abortion. This is a simple method, involving use of mifepristone with misoprostol as an abortifacient. The combination of these drugs was approved for medical abortion in India in April 2002. Combined with appropriate counseling and supervision, it is safe and acceptable for termination of pregnancy. Since April 2002, various doses of the drugs and routes of administration have been tried and tested. However, the ideal route and dose are yet to be determined. Vaginal administration of misoprostol minimizes gastrointestinal side effects and is more effective locally in the uterus. A strictly supervised procedure and several visits to the clinic are barriers to acceptability of this method. However, lately, self-administration of the drug with telephone evaluation of symptoms and less frequent hospital visits has improved its acceptability. The aim of this study was to ensure more effective 
utilization of this important advancement to maximize the reproductive health of women and maintain women's right to high quality health care services.

\section{Methods}

This prospective cross-sectional study was conducted in women seeking termination of pregnancy up to 59 days of gestation and was carried out between January 2007 and May 2008 in the Green Cross Hospital and Sakriti Clinic in Lucknow, India. The women in the study were counseled to help them decide on the method of abortion. As required by the MTP Act, patients were identified and gestational age was confirmed by scanning. A total of 160 patients volunteered to participate in this single-visit treatment study, and written informed consent was obtained from every patient. An emergency contact number and instructions were given to each patient as per protocol. The first $200 \mu \mathrm{g}$ dose of oral mifepristone was administered to the patients in the outpatient department followed by vaginal administration of $600 \mathrm{mg}$ of misoprostol by the patient at home or by hospital staff six hours later. All patients were instructed to report onset of bleeding/abortion, side effects, and discontinuation of medication by telephone, and these issues were then managed accordingly. A follow-up visit was scheduled on the 14th day after administration of the first dose. To confirm complete abortion, scanning was performed. Every patient filled out a questionnaire regarding choice of method and satisfaction level before and after the abortion. In addition, they also underwent counseling for family planning. The primary outcome was success, ie, complete abortion, after the first dose of vaginal misoprostol and the secondary outcome was complete abortion after the second dose.

\section{Results}

The mean age and parity of the patients was 26.28 years and one, respectively. Of the 160 women, $90 \%$ were married and $10 \%$ were unmarried. In addition, $49.4 \%$ patients had one child, while $27.5 \%$ had two or more children. Primigravida patients comprised $23.15 \%$ of the total study population. Thirty-five percent of the women had undergone termination of pregnancy previously $(23.85 \%$ once and $11.3 \%$ more than once). The proportion of women at weeks 7-8 of gestation was $65 \%$, while those at $\leq 6$ weeks of gestation comprised $10.6 \%$ (Table 1 ).

The success rate in terms of complete abortion was $98.12 \%$, with $1.25 \%$ of patients aborting after the second vaginal dose of misoprostol $400 \mu \mathrm{g}$. The failure rate was
Table I Participants characteristics $(n=160)$

\begin{tabular}{lll}
\hline Age (years) & Number & Percentage \\
\hline $20-25$ & 74 & $46.25 \%$ \\
$25-30$ & 50 & $31.25 \%$ \\
$30-35$ & 27 & $16.87 \%$ \\
$35-40$ & 9 & $5.62 \%$ \\
Mean age & 26.28 years & \\
Married & 144 & $90 \%$ \\
Unmarried & 16 & $10 \%$ \\
Parity & & \\
0 & 44 & $27.50 \%$ \\
I & 79 & $49.37 \%$ \\
$>2$ & 37 & $23.13 \%$ \\
Mean parity & 1 & \\
Prior termination of pregnancy & & \\
I & 28 & $23.8 \%$ \\
$>1$ & 18 & $11.3 \%$ \\
Gestational age & & \\
$<6$ weeks & 17 & $10.6 \%$ \\
6 weeks & 39 & $24.4 \%$ \\
7 weeks & 51 & $31.9 \%$ \\
8 weeks & 53 & $33.1 \%$ \\
\hline
\end{tabular}

$1.88 \%$, with $0.65 \%$ requiring surgical evacuation. In $86.9 \%$ of patients, vaginal bleeding began within 12 hours of vaginal misoprostol administration, while in $13.1 \%$ women bleeding began 12 hours after administration (Table 2).

\section{Discussion}

The combination of mifepristone and misoprostol has been shown to be effective for early termination of pregnancy of up to 63 days' gestation. ${ }^{2,3}$ There are various regimens and routes for medical abortion. The usual regimen is the administration of mifepristone followed by oral misoprostol 36-48 hours later. ${ }^{4,5,13}$ Previous studies have revealed that the success rate of complete abortion is very similar in cases where mifepristone doses of 200-600 $\mu \mathrm{g}$ are administered. ${ }^{8}$ Hence, we used the lower dose $(200 \mu \mathrm{g})$ of mifepristone in our study, followed by administration of a $600 \mathrm{mg}$ misoprostol vaginal tablet six hours later. Vaginal

Table 2 Outcome of medical abortion

\begin{tabular}{lll}
\hline Outcome & Number & Percentage \\
\hline Success rate & 157 & $98.12 \%$ \\
Primary & 155 & $96.87 \%$ \\
Secondary & 2 & $1.25 \%$ \\
Failure rate & 3 & $1.88 \%$ \\
Surgical intervention & 1 & $0.65 \%$ \\
Vaginal bleeding & 139 & $86.9 \%$ \\
$<12$ hours & 21 & $13.1 \%$ \\
$>12$ hours & & \\
Not confirmed by ultrasound & 36 & $22.5 \%$ \\
Confirmed by ultrasound & 124 & $77.5 \%$ \\
\hline
\end{tabular}


administration of misoprostol is highly effective because its bioavailability is route-dependent. ${ }^{9}$ By evaluating the questionnaires filled out by the patients in this study before and after abortion, we found that the percentage of women who had previously terminated pregnancy, either by the surgical or medical method, was $35.1 \%$, and $18 \%$ of these women preferred this modified regimen. This finding indicates that, given a choice, patients deem surgical abortion to be unacceptable. ${ }^{6,7}$ Furthermore, $92 \%$ of our patients chose the medical method offered to avoid hospitalization and because they preferred a noninvasive, natural method, such as a heavy menstrual period. No loss of work, complete privacy and confidentiality, and convenience were the other reasons cited for their preference. ${ }^{6,11}$ In our study, the success rate, ie, complete abortion, was $98.1 \%$, which is comparable with that reported in other studies. ${ }^{7,10}$ However, our findings do not coincide with those of another similar study. ${ }^{13}$ In that study, administration of vaginal tablets six hours after the first dose on the same day was not effective. However, the bioavailability of vaginal misoprostol is three times higher than that of oral misoprostol; hence, the rate of incomplete abortion is lower when misoprostol is administered via the vaginal route than when it is administered via the oral route. ${ }^{5}$ The $1.88 \%$ incomplete abortion rate in the current study is comparable with that reported in other studies. ${ }^{10}$ The decrease in efficacy with increasing gestational age ${ }^{11}$ may be the reason why $1.25 \%$ patients required a second dose of misoprostol. Our results are better than those of a previous study, ${ }^{12}$ and only one patient required surgical evacuation because of excessive vaginal bleeding on the second day after administration of the treatment dose. The mean abortion interval was 7.2 hours, which is comparable with that reported in a similar study. ${ }^{13}$ Side effects, such as nausea, vomiting, diarrhea, abdominal pain, and fever, are dose- and routedependent, ${ }^{10}$ although vaginal administration of the drugs enhances their effects in the reproductive tract and minimizes their effects in the gastrointestinal tract. No such side effects were noted in this study, except for mild abdominal cramps and prolonged bleeding beyond 14 days. The most common disadvantages of our study method were prolonged spotting for more than 14 days, as observed in four patients $(2.5 \%)$, and the time lag between administration of the drugs and complete abortion. ${ }^{6}$ The procedure acceptability of $96.2 \%$ was better than that in another study. ${ }^{6}$ Ninety-three percent of patients preferred this method, would undergo the same procedure if needed again in the future, and will recommend it to others. There were several reasons for this preference, ie, no hospitalization, noninvasive procedure employing natural methods (heavy menstruation), confidentiality, privacy, convenience, no absence from work, and no frequent hospital visits. ${ }^{6}$ We conclude from our study that, because women do not need frequent hospital visits and strict supervision, they can administer the vaginal dose of the treatment regimen by themselves at home, and telephone evaluation of symptoms with a single consultation visit is appropriate for follow-up. Furthermore, as stated in other studies, home-based medical abortion under limited supervision is an acceptable and effective method for early termination of pregnancy. ${ }^{14}$ Further study of this method is now needed in a larger population of women.

\section{Conclusion}

Medical termination of pregnancy is a promising alternative to surgical abortion. In our study, women given the options to choose were certain about this method. Only a single visit was required for treatment, and the follow-up visit was replaced by telephone evaluation of symptoms, which is acceptable in terms of safety. This method is highly preferred and efficacious, and maintains women's right to high quality health care services. This method for abortion promises to revolutionize reproductive health care, especially in developing countries, which would also benefit from awareness about the dangers of unsafe abortions and the availability of safe abortion services.

\section{Disclosure}

The author reports no conflict of interest in this work.

\section{References}

1. Ministry of Health and Family Welfare (MOHFW). National Population Policy, 2000. New Delhi: Government of India; 2000.

2. World Health Organization. Termination of pregnancy with reduced doses of mifepriston. Br Med J. 1993;307:532-537.

3. Baird DT, Sukcharoen N, Thong KJ. Randomized trial of misoprostol and cervagem in combination with a reduced dose of mifepriston for induction of abortion. Hum Reprod. 1995;10:1521-1527.

4. Child TJ, Thomas J, Rees M, Mackenzie IZ. A comparative study of surgical and medical procedures: 932 pregnancy terminations up to 63 days gestation. Hum Reprod. 2001;16:67-71.

5. El-Refaey H, Rajasekar D, Abdalia M, Calder L, Templeton A. Induction of abortion with mifepriston (RU 486) and oral or vaginal misoprostol N Engl J Med. 1995;332:983-987.

6. Fiala C, Winikoff B, Helstrom L, Hellborg M, Gemzell-Danielsson K Acceptability of home-use of misoprostol in medical abortion. Contraception. 2004;70:387-392.

7. Guengant JP, Bangou J, Elul B, Ellertson C. Mifepriston-misoprostol medical abortion: Home administration of misoprostol in Guadeloupe. Contraception. 1999;60:167-172.

8. Lyons M. Mifepriston dose in the regimen for medical abortion Contraception. 2006;74:21-25.

9. Pymar HC, Cretin MD, Schwartz JL. Mifepriston followed on the same day by vaginal misoprostol for early abortion. Contraception 2001;64:87-92. 
10. Schaff EA, Eisinger SH, Stadalius LS, Franks P, Gore BZ, Poppema S. Low-dose mifepriston $200 \mathrm{mg}$ and vaginal misoprostol for abortion. Contraception. 1999;59:1-6.

11. Schreiber CA, Cretin MD, Harwood B, Murthy AS. A pilot study of mifepriston, mefipriston, and misoprostol administered at the same time for abortion in women with gestation from 50 to 63 days. Contraception. 2005;71:447-450.

12. Kapp N, Borgatta L, Ellis SC, Stubblefield P. Simultaneous very low dose mifepriston and vaginal misoprostol for medical abortion. Contraception. 2006;73:525-527.
13. Guest J, Chien P, Thomson M, Kosseim ML. Randomised controlled trial comparing efficacy of same day administration of mifepriston and misoprostol for termination of pregnancy with the standard 36- to 48-hour protocol. BJOG. 2005;112:1457.

14. Harper C, Ellertson C, Winikoff B. Could American women use mifepriston-misoprostol pills safely with less medical supervision? Contraception. 2002;65:133-142.

\section{Publish your work in this journal}

Open Access Journal of Contraception is an international, peerreviewed, open access, online journal, publishing original research, reports, reviews and commentaries on all areas of contraception. In addition to clinical research, demographics and health-related aspects, the journal welcomes new findings in animal and preclinical studies relating to understanding the biological mechanisms and practical development of new contraceptive agents. The manuscript management system is completely online and includes a very quick and fair peer-review system. Visit http://www.dovepress.com/testimonials.php to read real quotes from published authors. 